\title{
Defining growth in small pulmonary nodules using volumetry: results from a "coffee-break" CT study and implications for current nodule management guidelines
}

\author{
Emily C. Bartlett ${ }^{1,2} \cdot$ Samuel V. Kemp ${ }^{2,3} \cdot$ Bhavin Rawal $^{1,2} \cdot$ Anand Devaraj $^{1,2}$
}

Received: 20 May 2021 / Revised: 10 August 2021 / Accepted: 24 August 2021 / Published online: 27 September 2021

(c) The Author(s) 2021

\begin{abstract}
Objectives An increase in lung nodule volume on serial CT may represent true growth or measurement variation. In nodule guidelines, a $25 \%$ increase in nodule volume is frequently used to determine that growth has occurred; this is based on previous same-day, test-retest (coffee-break) studies examining metastatic nodules. Whether results from prior studies apply to small non-metastatic nodules is unknown. This study aimed to establish the interscan variability in the volumetric measurements of small-sized non-metastatic nodules.

Methods Institutional review board approval was obtained for this study. Between March 2019 and January 2021, 45 adults ( 25 males; mean age 65 years, range $37-84$ years) with previously identified pulmonary nodules $\left(30-150 \mathrm{~mm}^{3}\right.$ ) requiring surveillance, without a known primary tumour, underwent two same-day CT scans. Non-calcified solid nodules were measured using commercial volumetry software, and interscan variability of volume measurements was assessed using a Bland-Altman method and limits of agreement.

Results One hundred nodules (range $28-170 \mathrm{~mm}^{3}$; mean $81.1 \mathrm{~mm}^{3}$ ) were analysed. The lower and upper limits of agreement for the absolute volume difference between the two scans were $-14.2 \mathrm{~mm}^{3}$ and $12.0 \mathrm{~mm}^{3}$ respectively (mean difference 1.09 $\mathrm{mm}^{3}$, range $-33-12 \mathrm{~mm}^{3}$ ). The lower and upper limits of agreement for relative volume difference were $-16.4 \%$ and $14.6 \%$ respectively (mean difference $0.90 \%$, range $-24.1-32.8 \%$ ).

Conclusions The interscan volume variability in this cohort of small non-metastatic nodules was smaller than that in previous studies involving lung metastases of varying sizes. An increase of $15 \%$ in nodule volume on sequential CT may represent true growth, and closer surveillance of these nodules may be warranted.

Key Points

- In current pulmonary nodule management guidelines, a threshold of $25 \%$ increase in volume is required to determine that true growth of a pulmonary nodule has occurred.

- This test-retest (coffee break) study has demonstrated that a smaller threshold of $15 \%$ increase in volume may represent true growth in small non-metastatic nodules.

- Closer surveillance of some small nodules growing 15-25\% over a short interval may be appropriate.
\end{abstract}

Keywords Multiple pulmonary nodules $\cdot$ Lung neoplasms $\cdot$ Growth $\cdot$ Cancer screening

Emily C. Bartlett

E.Bartlett@rbht.nhs.uk; Emily.Bartlett@nhs.net

1 Department of Radiology, Royal Brompton Hospital, Sydney Street, London SW3 6NP, UK

2 National Heart and Lung Institute, Imperial College, London SW3 6LY, UK

3 Department of Respiratory Medicine, Royal Brompton Hospital, Sydney Street, London SW3 6NP, UK

$\begin{array}{ll}\text { Abbreviations } \\ \text { BTS } & \text { British Thoracic Society } \\ \text { CT } & \text { Computed tomography } \\ \text { LDCT } & \text { Low-dose computed tomography } \\ \text { NELSON } & \text { Nederlands-Leuvens Longkanker Screenings } \\ & \text { Onderzoek (Trial) } \\ \text { PACS } & \text { Picture Archiving and Communication System } \\ \text { V } & \text { Volume } \\ \text { VDT } & \text { Volume doubling time }\end{array}$




\section{Introduction}

Lung nodule growth is defined as an increase in nodule diameter or volume on sequential computed tomography (CT) scans and is a powerful predictor of lung malignancy $[1,2]$. Measuring nodule growth can be performed using electronic callipers or semi-automated tools. However, establishing that true nodule growth has occurred can be challenging in cases where the increase in nodule size is small, due to the inherent limitations of measurement tools [3]. For this reason, many clinical practice guidelines stipulate that a minimum increase in nodule size must be achieved before nodule growth can be determined. For example, the Fleischner Society guidelines stipulate that a threshold of 2-mm diameter growth should be used to define true nodule growth [4]. By contrast, the British Thoracic Society (BTS) nodule management guidelines stipulate a $25 \%$ increase in nodule volume to determine growth [5], a threshold also used in the Nederlands-Leuvens Longkanker Screenings Onderzoek (NELSON) lung cancer screening trial $[6,7]$.

The threshold of a $25 \%$ increase in volume to determine true nodule growth in the BTS guidelines and NELSON nodule management protocol was based on a number of in vivo "coffee break" studies, in which individuals were scanned twice on the same day, demonstrating up to $25 \%$ variability in nodule volumes [8-10]. This variability has been attributed to slight differences in nodule segmentation, including for nodules which are irregular in shape, or attached to pleural surfaces or vessels [9], and to patient factors, such as the level of patient inspiration or pulsation of the heart [10].

However, results from previous so-called coffee break studies may not necessarily be applicable to smaller nodules detected incidentally or in lung cancer screening. This is because prior studies have mainly involved participants with known lung metastases ranging in size from $<10$ to $5000 \mathrm{~mm}^{3}$. Lung metastases are known to be more likely to be smooth in outline and spherical in shape than incidentally or screen-detected nodules, and the interscan variability of such nodules has previously been demonstrated to be less than irregular and non-spherical nodules [8]. Furthermore, small nodules might be expected to show greater variability in nodule volumes than larger nodules due to their greater surface area-to-volume ratio [3].

The purpose of this study was therefore to determine the interscan variability in nodule volume measurements and to establish whether the $25 \%$ threshold to determine true growth $[5,6]$ was applicable in the setting of small non-metastatic pulmonary nodules.

\section{Materials and methods}

Institutional review board approval was obtained for this study. Written consent was obtained from all recruited participants.

Seventy adults who were due to attend a scheduled clinically required follow-up at the Royal Brompton Hospital for either screen- or incidentally detected non-metastatic, solid, non-calcified, small-sized lung nodules $<150 \mathrm{~mm}^{3}$ between March 2019 and January 2021 were invited by letter to participate in this study. Written informed consent was obtained from forty-nine adults who agreed to participate in this study.

All 49 participants underwent an initial low-dose CT (LDCT) scan (scan 1) for clinical follow-up. Scans were immediately reviewed by a thoracic radiologist (with 3 years of experience in subspecialty thoracic radiology) to assess for the presence of the known lung nodule. In four participants, the nodule previously identified had resolved $(n=2)$ or appreciably reduced in size to $<30 \mathrm{~mm}^{3}(n=2)$; these participants were therefore not recruited to the study, leaving 45 participants ( 25 males, median age 65 years, range $37-84$ years) in the final study cohort. These individuals left the CT table after the first scan (scan 1), and subsequently during the same appointment were repositioned on the CT table, and scanned a second time (scan 2) after a short interval (2-13 min) using identical scanning parameters.

\section{CT image acquisition and transfer}

All participants were scanned on a 128-slice Siemens Somatom Definition Edge multidetector CT scanner (Siemens Healthineers). Participants were scanned in a supine position from the lung apices to the lung bases, with a breath hold, and without the administration of contrast medium. Weightbased scanning parameters were used such that tube voltage was pre-set and selected for patient weight $(<50 \mathrm{~kg}$ at $100 \mathrm{kV}$, $50-<80 \mathrm{~kg}$ at $120 \mathrm{kV}$, and $\geq 80 \mathrm{~kg}$ at $140 \mathrm{kV})$. Automatic exposure control (Care Dose4D) was enabled, resulting in adaptation of the tube current (mAs) based on patient size. Scanning was performed with a gantry rotation time of $500 \mathrm{~ms}$. Images were reconstructed using filtered back projection with a soft tissue reconstruction kernel (B30f) [11], with a slice thickness/increment of $1 \mathrm{~mm} / 0.7 \mathrm{~mm}$. Images were transferred to the local PACS system (Impax, Agfa Healthcare).

\section{CT reading}

Semi-automated nodule volumetric analysis was performed using SyngoVia ${ }^{\mathrm{TM}}$ software (Syngo.Via Client 5.1, 
SyngoVia ${ }^{\mathrm{TM}}$, Siemens Healthineers). Images were displayed in the SyngoVia ${ }^{\mathrm{TM}}$ software with a window width of $1500 \mathrm{HU}$ and level of $-500 \mathrm{HU}$. To assess interscan variability, all CT reading was performed by one radiologist (E.B.). Nodules were selected by drawing a line across the nodule using a mouse and were automatically segmented and measured by the software. No manual correction of volumetric measurements was performed; if segmentation was judged to be poor, for example because of attachment to an adjacent vessel or the pleura, nodules were excluded from analysis. Saved images showing measured nodule volumes were sent to the local PACS system. Nodules were characterised as being spherical or non-spherical. Non-spherical nodules were further sub-categorised as having a smooth or an irregular margin. All nodules in the range $30-150 \mathrm{~mm}^{3}$ were included in this study. Nodules smaller than $30 \mathrm{~mm}^{3}$ were excluded from analysis, as such nodules would not ordinarily warrant follow-up in clinical practice or in the screening setting after either a baseline or incident round scan. Larger nodules $>150 \mathrm{~mm}^{3}$ are approaching a size threshold for intervention or further investigation $\left(>200 \mathrm{~mm}^{3}\right)$ in several screening protocols and have also been studied extensively previously, and thus were not evaluated.

As a secondary analysis, inter-observer variability in nodule volume measurements was assessed. The recorded nodules volumes for the first observer (E.B.) were deleted from the Syngovia ${ }^{\mathrm{TM}}$ volumetry platform. Following this, a second observer, with 3 years thoracic radiology experience (B.R.), independently re-measured and recorded the nodule volumes on the first of the two scans performed, blinded to the measurements of the first observer. If a nodule was felt to be poorly segmented by the software by the second observer, this was also recorded.

\section{Statistical evaluation}

The inter-scan variability of volumetric nodule measurements was assessed using the Bland-Altman method of assessing agreement $[12,13]$. Since the true volume of any given nodule is not known, the mean of the two measured volumes $\left(V_{1}-V_{2} / 2, V_{\text {mean }}\right)$ is assumed to represent the true nodule volume. In line with previous studies, the absolute difference in the measured nodule volumes $\left(V_{1}-V_{2}, \mathrm{~mm}^{3}\right)$ is plotted against the mean nodule volume $[8,10,14-16]$. The difference in nodule volume is also shown as a proportion of the mean nodule volume $\left(\left(V_{1}-V_{2} / V_{\text {mean }}\right) \times 100, \%\right)$, to provide a measure of the relative difference in volumes between the two scans. The upper and lower limits of agreement are calculated as the range of $95 \%$ of the observed absolute and relative differences in the two volume measurements (1.96 standard deviations above and below the mean difference). A sub-analysis was also performed of the limits of agreement for the smallest nodules, $30-<80 \mathrm{~mm}^{3}$, compared to larger nodules $80-150 \mathrm{~mm}^{3}$.

To assess interobserver variability of nodule measurements, the same methods were used to establish the upper and lower limits of agreement in the absolute and relative volume measurements of the 100 nodules measured by both observers.

All statistics were carried out in SPSS version 26 and 28 (IBM SPSS Statistics for Windows).

\section{Results}

A total of 107 small-sized nodules were identified in 45 participants undergoing two LDCT scans. Four nodules were $>150 \mathrm{~mm}^{3}$ on the volumetric measurements on both the initial and second scans, and one nodule was just under $30 \mathrm{~mm}^{3}$ on both scans. The first observer recorded that two nodules were poorly segmented by the volumetry software; one was irregular in contour and juxtavascular, and the second nodule was irregular but not abutting the vasculature or pleura. All seven nodules were excluded from analysis. Therefore, a total of 100 non-calcified nodules in 41 participants were evaluated (Fig. 1). The demographic characteristics of the 41 study participants in whom nodules were finally evaluated, as well as the characteristics of the nodules examined, are given in Table 1.

Nodule volumes on scan 1 ranged from 31 to $158 \mathrm{~mm}^{3}$ (mean $80.5 \mathrm{~mm}^{3}$, SD $36.5 \mathrm{~mm}^{3}$ ), and on scan 2 from 28 to $170 \mathrm{~mm}^{3}$ (mean $81.6 \mathrm{~mm}^{3}$, SD $37.6 \mathrm{~mm}^{3}$ ) (Fig. 2).

The mean absolute volume difference between the two scans was $-1.09 \mathrm{~mm}^{3}$ (95\% confidence interval -2.42 to $0.24)$, range -33 to $12 \mathrm{~mm}^{3}$. The lower and upper limits of agreement were $-14.2 \mathrm{~mm}^{3}$ and $12.0 \mathrm{~mm}^{3}$ (Fig. 3a). The mean relative difference in nodule volumes was $-0.90 \%$, range -24.1 to $32.8 \%$. The lower and upper limits of agreement were -16.4 and $14.6 \%$ respectively (Fig. $3 b$ ). Lower and upper limits of agreement for the relative difference in nodule volumes for 58 nodules with a mean volume of $30-<80 \mathrm{~mm}^{3}$ were -16.8 to $16.2 \%$, with a mean relative volume difference of $-0.3 \%$ ( $95 \%$ confidence interval -2.51 to $1.91 \%$ ). For the 42 larger nodules, $80-150 \mathrm{~mm}^{3}$, the upper and lower limits of agreement for the relative difference in nodule volumes were -15.8 to $12.3 \%$, with a mean relative volume difference of $-1.7 \%$ (95\% confidence interval -3.96 to $0.50 \%$ ).

Identical nodule measurements were made by the second observer in 95/100 nodules measured. The second observer determined that one additional nodule was poorly segmented by the volumetry software, but otherwise agreed with the first observer, that the remaining 99 nodules measured were well segmented. The mean absolute volume difference between the measurements of the two observers of the 100 
Fig. 1 Flow diagram of participant inclusion

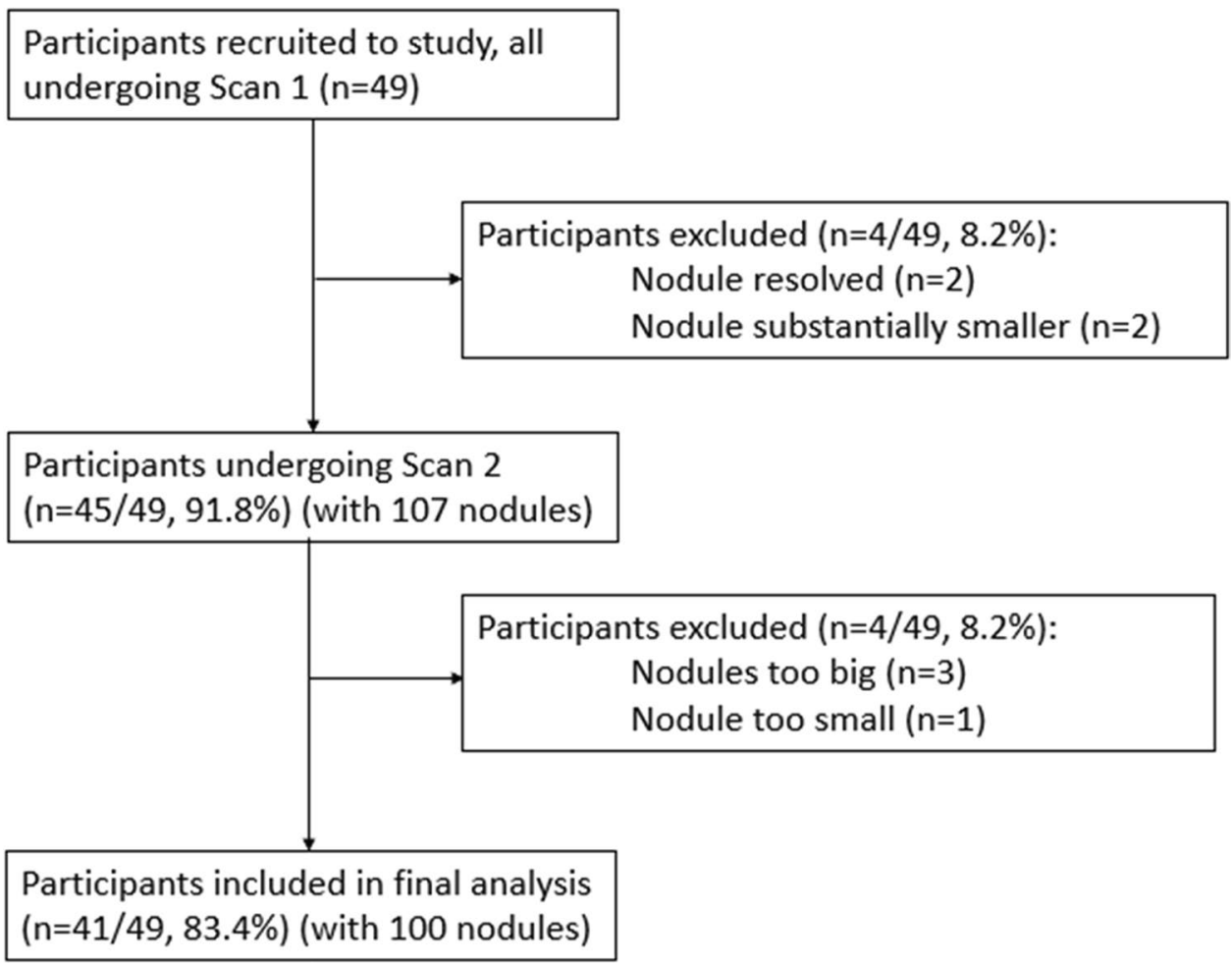

Table 1 Demographic characteristics of 41 participants included in final analysis, with nodule characteristics of 100 evaluated nodules

\begin{tabular}{ll}
\hline Study participants-demographics & \\
\hline Age (years) (median, range) & $67.2(37-84)$ \\
Sex (no. of patients, $\%)$ & \\
Male & $23(56.1 \%)$ \\
Female & $18(43.9 \%)$ \\
Nodule characteristics & \\
Total number of nodules & 100 \\
Lobe (no. of nodules, $\%)$ & \\
Right upper lobe & $26(26 \%)$ \\
Right middle lobe & $6(6 \%)$ \\
Right lower lobe & $21(21 \%)$ \\
Left upper lobe & $21(21 \%)$ \\
Left lower lobe & $26(26 \%)$ \\
Nodule morphology (no. of nodules, $\%)$ & \\
Spherical, smooth margin & $28(28 \%)$ \\
Non-spherical/polygonal, smooth margin & $7(7 \%)$ \\
Non-spherical, irregular margin & $65(65 \%)$ \\
Nodule location & \\
Freestanding intraparenchymal nodules & $80(80 \%)$ \\
Juxtapleural nodules & $2(2 \%)$ \\
Perifissural nodules & $5(5 \%)$ \\
Juxtavascular nodules & $13(13 \%)$ \\
\hline
\end{tabular}

nodules was $-0.39 \mathrm{~mm}^{3}$ ( $95 \%$ confidence interval -0.85 to 0.73 ), range -20 to $2 \mathrm{~mm}^{3}$. The lower and upper limits of agreement were -4.96 to $4.18 \mathrm{~mm}^{3}$ respectively. The mean relative volume difference between the measurements of the two observers was $-0.03 \%$ (95\% confidence interval -0.38 to 0.43 ), range -12.1 to $14.8 \%$. The lower and upper limits of agreement for the relative difference in nodule volumes measured by the two observers were -3.98 to $4.01 \%$.

In view of the wider limits of agreement for the smaller nodules $<80 \mathrm{~mm}^{3}$ than the larger nodules $80-150 \mathrm{~mm}^{3}$, a post hoc analysis was performed using linear regression to assess for proportional bias. The values of the relative difference in nodule volumes (\%) were converted to absolute values (removing negative signs) and regressed against the mean nodule volume [17]. This demonstrated no significant relationship between the relative difference in nodule volume and the mean nodule volume $(p=0.66)$.

Current nodule management guidelines assume that an increase in volume of $<25 \%$ represents stability $[5,7,18]$. Given that we found that the relative volume difference was approximately $15.5 \%$ either side of the mean nodule volume, and therefore that any increase above this may represent true growth, we performed a further post hoc analysis to model how these results impact current nodule management guidelines for small nodules (Table 2). Specifically, we modelled the impact of assuming that an increase of 15-24\% represented true growth. Furthermore, we assumed that the optimal threshold for intervention and further investigation 
Fig. 2 Volumetric measurements of 100 nodules on scans 1 and 2

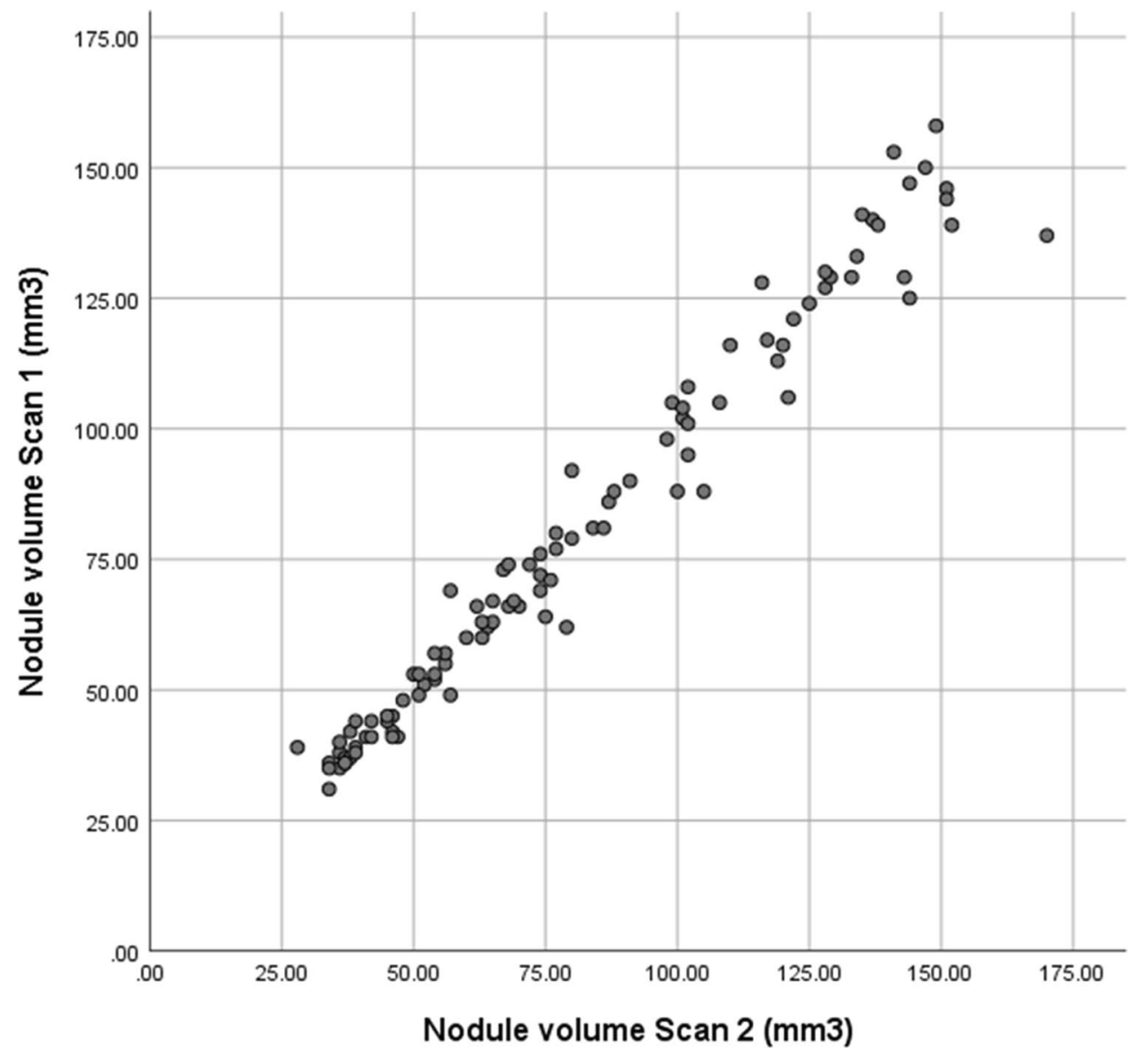

in growing small nodules was $200 \mathrm{~mm}^{3}$ [18-21]. As shown in Table 2, the assumption of nodule stability if a nodule is growing $<25 \%$ but $>15 \%$ between serial scans may results in a short delay to further investigation for some small nodules.

\section{Discussion}

This study sought to establish the reproducibility of volumetric software in measuring small non-metastatic pulmonary nodules $<150 \mathrm{~mm}^{3}$, using established methods. Given the effects of partial voluming on CT, and the non-spherical nature of many nodules detected incidentally in the clinical and lung screening setting, the limits of agreement were expected to be wider than published in previous similar studies involving metastatic nodules. Unexpectedly, this study demonstrated narrower limits of agreement, $15.5 \%$ either side of the mean relative difference in nodule volume. The upper and lower limits of agreement for the relative difference in nodule volumes measured by two observers were only $\pm 4 \%$, confirming data from previous studies that interobserver variability in nodule volume measurements contributes very little towards the overall variability in nodule measurements $[9,10]$.

Compared to previous similar studies where the morphological characteristics of nodules have been compared $[8,10]$, a greater proportion of the nodules in the current study were irregular in contour, as was predicted due to the non-metastatic nature of nodules included in this study. Despite this, the current study has shown narrower limits of agreement for the relative nodule volume measurements than the majority of previous studies $[8-10,15,16]$. This may reflect advances in volumetry software over the past 15 years, differences in volumetry software packages, and advances in CT scanner technology. One study by Zhao et al demonstrated narrower $95 \%$ limits of agreement $(-12.1$ to $+13.4 \%)$ in the volumetric measurements of a cohort of 32 known non-small-cell lung cancers; however, the mean tumour size in this study was $>3-\mathrm{cm}$ diameter, and results are therefore not directly comparable to the current study of very small lung nodules [22]. The impact of nodule size on measurement variability has been studied in a small number of previous in vivo studies with conflicting results $[8-10,15]$. Wormanns et al found very similar limits of agreement in nodules $\leq 10 \mathrm{~mm}$ in diameter, compared to those $>10 \mathrm{~mm}$ in diameter [9], whereas 
Fig. 3 a Absolute volume difference $\left(\mathrm{mm}^{3}\right)$ between volume measurements on the first and second scans, plotted against the mean nodule volume $\left(\mathrm{mm}^{3}\right)$. Solid line demonstrates the mean absolute volume difference, and dashed lines show the upper and lower limits of agreement. b Relative difference in nodule volumes $(\%)$ plotted against the mean nodule volume $\left(\mathrm{mm}^{3}\right)$. The solid line demonstrates the mean relative volume difference, and dashed lines show the upper and lower limits of agreement
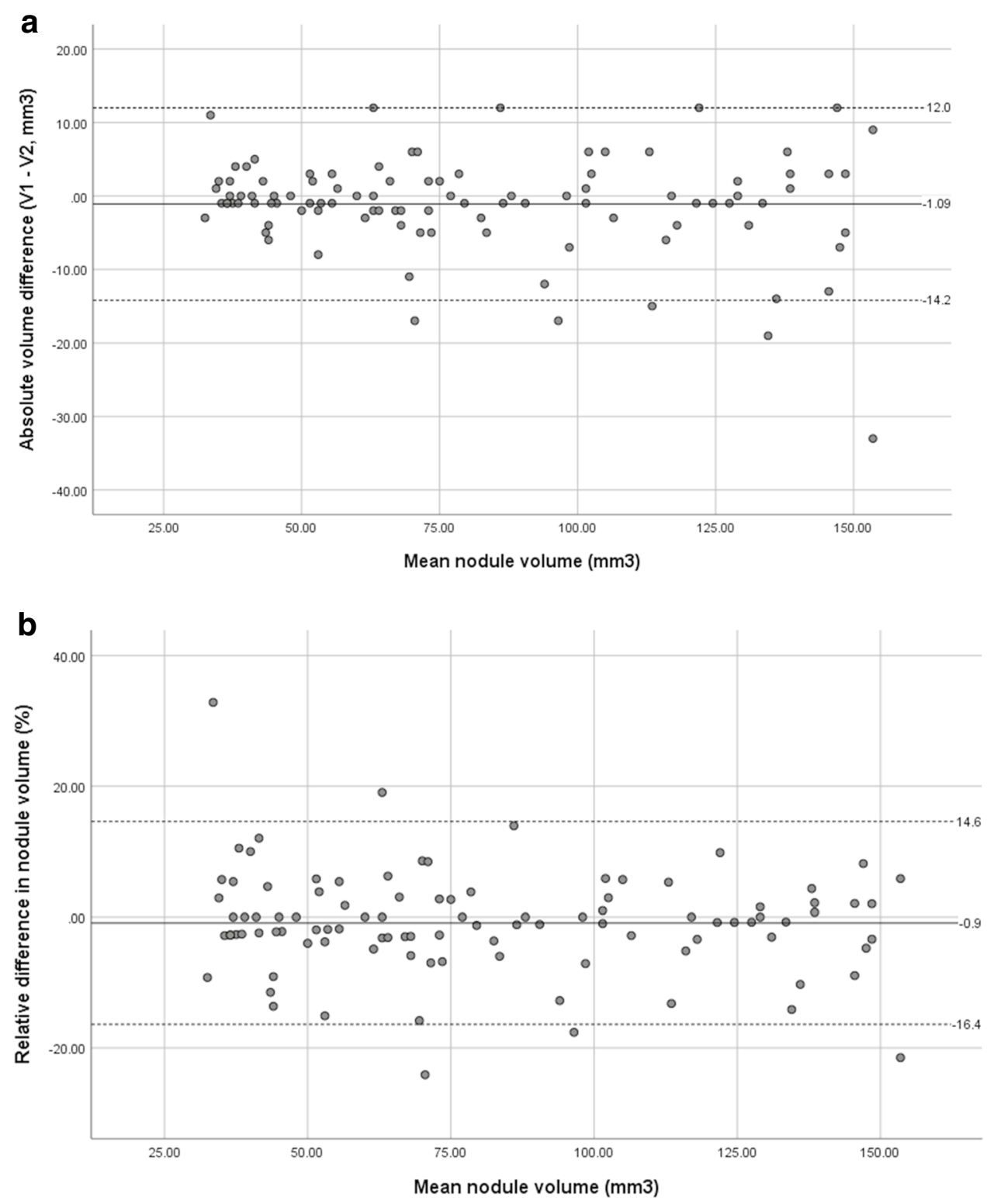

Goodman et al found that confidence limits narrow with increasing nodule volume [10]. Comparing the volumetric measurements of metastatic nodules $<8 \mathrm{~mm}$ and $\geq 8 \mathrm{~mm}$ in diameter, De Hoop et al found that the interscan variability in nodule volumes decreased with increasing nodule volume when measured by 5 of 6 nodule volumetry software packages [16]. By contrast, Talwar and colleagues found lower variability for nodule measurements in nodules $<500 \mathrm{~mm}^{3}$ compared to those over $500 \mathrm{~mm}^{3}$ [15]. However, no previous studies have focussed specifically on small nodules $<150 \mathrm{~mm}^{3}$ (equivalent to $<6.59-\mathrm{mm}$ diameter), frequently encountered in both the lung cancer screening setting, and incidentally on CT scans of the chest. This study demonstrated no evidence that the relative difference in nodule volumes (\%) varies in relation to the absolute size of the nodule for small nodules between 30 and $150 \mathrm{~mm}^{3}$.

A number of nodule management guidelines in the clinical and screening setting recommend a 3- or 6-month follow-up CT for solid nodules in the size range $30-150 \mathrm{~mm}^{3}[5,7$, $19,20]$, the lower limit applying to new nodules developing on incident round screening CTs [23]. Results from this study indicate that $>15 \%$ growth in a nodule volume may represent true nodule growth in this cohort of small nodules, and that an assumption of stability should not be made for nodules growing 15-25\% over 3-6 months. This assumption of stability may result in a short delay to investigation of a cohort of nodules measuring $115-150 \mathrm{~mm}^{3}$, and these may require closer short-term surveillance, for example, with a repeat scan in a further 3-6 months, particularly as 


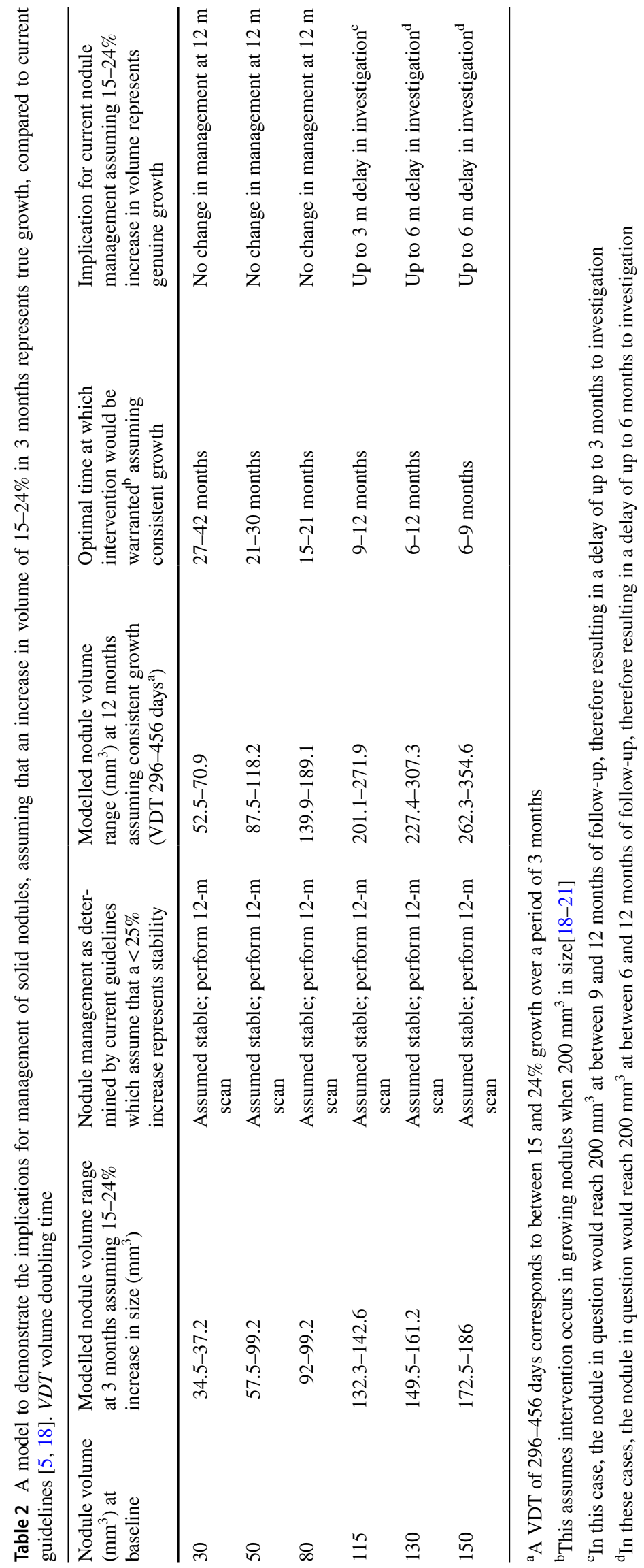


nodules approach a threshold for intervention $\left(>200 \mathrm{~mm}^{3}\right)$. This would prevent a potential delay to diagnosis of malignancy in growing nodules in this size range. Provided that a minimum volume threshold of $200 \mathrm{~mm}^{3}$ is maintained with nodule management protocols prior to intervention, little harm is expected to result from lowering the threshold to determine growth to $15 \%$ in this cohort of nodules $115-150 \mathrm{~mm}^{3}$, since at least one further short-term interval scan would be required to confirm persistent growth prior to any invasive procedure. This study did not evaluate nodules in the size range $150-300 \mathrm{~mm}^{3}$ which are also considered indeterminate in a number of nodule management protocols utilised in screening [5, 18-20,24]. As several previous studies have incorporated larger nodules and found wider limits of agreement (around $\pm 25 \%$ ), maintaining the threshold of $25 \%$ growth to confirm true growth may be appropriate for larger nodules to prevent potential over-investigation of nodules in the range $150-300 \mathrm{~mm}^{3}$. Further studies are warranted in this regard.

Strengths of the study include that all individuals were scanned with an identical low-dose scanning protocol on the same scanner, and analysis was performed using a modern volumetry software package. Therefore, many of the conditions in which this study was performed are likely to closely mirror conditions in a lung screening cohort. A limitation of the current study is that the results have been obtained using a single software package and therefore may not apply to other volumetry packages. Studies of similar patient cohorts using other software packages are warranted. It is noted that, in clinical practice, further variability may be introduced through several other variables including CT acquisition factors (such as dose), reconstruction techniques (filtered back projection or iterative reconstruction), and reconstruction parameters including slice thickness. Of these factors, reconstruction algorithm and slice thickness are the primary contributors to interscan variability [3]. As much as possible, all such parameters must be kept constant between scans comparing nodule volumes. In the event that scan acquisition and reconstruction parameters are not constant between scans, it may be more appropriate to use the $25 \%$ threshold for growth currently in use. However, in the screening setting, scans are ordinarily performed with identical scanning parameters and therefore the findings of our current study would apply in this context. It is also noted that the findings of this study should not be extrapolated to nodules which are poorly segmented by volumetry software and therefore measured by diameter. Such nodules may require ongoing surveillance for an extended period (up to 2 years) based on nodule diameter.

In conclusion, this study has demonstrated that, for small non-metastatic pulmonary nodules, true growth can be reliably concluded to have occurred with a volume change of $>15 \%$ where scanning parameters are identical between scans. Caution, therefore, should be exercised in participants with nodules growing $15-25 \%$, particularly those nodules in the range of $115-150 \mathrm{~mm}^{3}$. Under current nodule management guidelines widely used in the clinical and screening setting, such nodules would be presumed to be stable, and closer surveillance of these nodules may be warranted.

Funding The authors state that this work has not received any funding.

\section{Declarations}

Guarantor The scientific guarantor of this publication is Professor Anand Devaraj.

Conflict of interest Professor Devaraj declares personal fees from Brainomix, outside the submitted work.

Statistics and biometry No complex statistical methods were necessary for this paper.

Informed consent Written informed consent was obtained from all subjects (patients) in this study.

Ethical approval Institutional Review Board approval was obtained. Ethical approval for this study was granted by the NHS London Brent Research Ethics Committee.

\section{Methodology}

- prospective

- performed at one institution

Open Access This article is licensed under a Creative Commons Attribution 4.0 International License, which permits use, sharing, adaptation, distribution and reproduction in any medium or format, as long as you give appropriate credit to the original author(s) and the source, provide a link to the Creative Commons licence, and indicate if changes were made. The images or other third party material in this article are included in the article's Creative Commons licence, unless indicated otherwise in a credit line to the material. If material is not included in the article's Creative Commons licence and your intended use is not permitted by statutory regulation or exceeds the permitted use, you will need to obtain permission directly from the copyright holder. To view a copy of this licence, visit http://creativecommons. org/licenses/by/4.0/.

\section{References}

1. Horeweg N, Scholten ET, de Jong PA et al (2014) Detection of lung cancer through low-dose CT screening (NELSON): a prespecified analysis of screening test performance and interval cancers. Lancet Oncol 15(12):1342-1350

2. Henschke CI, Yankelevitz DF, Yip R et al (2012) Lung cancers diagnosed at annual CT screening: volume doubling times. Radiology 263(2):578-583

3. Devaraj A, van Ginneken B, Nair A, Baldwin D (2017) Use of volumetry for lung nodule management: theory and practice. Radiology 284(3):630-644 
4. Bankier AA, MacMahon H, Goo JM, Rubin GD, Schaefer-Prokop CM, Naidich DP (2017) Recommendations for Measuring pulmonary nodules at CT: a statement from the Fleischner Society. Radiology 285(2):584-600

5. Callister ME, Baldwin DR, Akram AR et al (2015) British Thoracic Society guidelines for the investigation and management of pulmonary nodules. Thorax 70(Suppl 2):ii1-ii54.

6. Horeweg N, van der Aalst CM, Vliegenthart R et al (2013) Volumetric computed tomography screening for lung cancer: three rounds of the NELSON trial. Eur Respir J 42(6):1659-1667

7. Xu DM, Gietema H, de Koning $\mathrm{H}$ et al (2006) Nodule management protocol of the NELSON randomised lung cancer screening trial. Lung Cancer 54(2):177-184

8. Gietema HA, Schaefer-Prokop CM, Mali WP, Groenewegen G, Prokop M (2007) Pulmonary nodules: interscan variability of semiautomated volume measurements with multisection CTinfluence of inspiration level, nodule size, and segmentation performance. Radiology 245(3):888-894

9. Wormanns D, Kohl G, Klotz E et al (2004) Volumetric measurements of pulmonary nodules at multi-row detector CT: in vivo reproducibility. Eur Radiol 14(1):86-92

10. Goodman LR, Gulsun M, Washington L, Nagy PG, Piacsek KL (2006) Inherent variability of CT lung nodule measurements in vivo using semiautomated volumetric measurements. AJR Am J Roentgenol 186(4):989-994

11. Wang Y, de Bock GH, van Klaveren RJ et al (2010) Volumetric measurement of pulmonary nodules at low-dose chest CT: effect of reconstruction setting on measurement variability. Eur Radiol 20(5):1180-1187

12. Bland JM, Altman DG (1986) Statistical methods for assessing agreement between two methods of clinical measurement. Lancet 1(8476):307-310

13. Patton N, Aslam T, Murray G (2006) Statistical strategies to assess reliability in ophthalmology. Eye (Lond) 20(7):749-754

14. Bland JM, Altman DG (1995) Comparing methods of measurement: why plotting difference against standard method is misleading. Lancet 346(8982):1085-1087

15. Talwar A, Willaime JMY, Pickup LC et al (2018) Pulmonary nodules: assessing the imaging biomarkers of malignancy in a “coffee-break”. Eur J Radiol 101:82-86
16. de Hoop B, Gietema H, van Ginneken B, Zanen P, Groenewegen G, Prokop M (2009) A comparison of six software packages for evaluation of solid lung nodules using semi-automated volumetry: what is the minimum increase in size to detect growth in repeated CT examinations. Eur Radiol 19(4):800-808

17. Ludbrook J (2010) Confidence in Altman-Bland plots: a critical review of the method of differences. Clin Exp Pharmacol Physiol 37(2):143-149

18. Oudkerk M, Devaraj A, Vliegenthart R et al (2017) European position statement on lung cancer screening. Lancet Oncol 18(12):e754-e766

19. Bartlett EC, Kemp SV, Ridge CA et al (2020) Baseline results of the West London lung cancer screening pilot study - impact of mobile scanners and dual risk model utilisation. Lung Cancer 148:12-19

20. Horst C, Dickson JL, Tisi S et al (2020) Delivering low-dose CT screening for lung cancer: a pragmatic approach. Thorax 75(10):831-832

21. Walter JE, Heuvelmans MA, Ten Haaf K et al (2019) Persisting new nodules in incidence rounds of the NELSON CT lung cancer screening study. Thorax 74(3):247-253

22. Zhao B, James LP, Moskowitz CS et al (2009) Evaluating variability in tumor measurements from same-day repeat CT scans of patients with non-small cell lung cancer. Radiology 252(1):263-272

23. Walter JE, Heuvelmans MA, Oudkerk M (2017) Small pulmonary nodules in baseline and incidence screening rounds of low-dose CT lung cancer screening. Transl Lung Cancer Res 6(1):42-51

24. Crosbie PA, Gabe R, Simmonds I et al (2020) Yorkshire Lung Screening Trial (YLST): protocol for a randomised controlled trial to evaluate invitation to community-based low-dose CT screening for lung cancer versus usual care in a targeted population at risk. BMJ Open. 10(9):e037075

Publisher's note Springer Nature remains neutral with regard to jurisdictional claims in published maps and institutional affiliations. 